\title{
A recurrent fusion gene in high-grade endometrial stromal sarcoma: a new tool for diagnosis and therapy?
}

Thomas Ried ${ }^{1 *}$ and Timo Gaiser ${ }^{2 *}$

\begin{abstract}
High-grade endometrial stromal sarcomas (ESSs) are an aggressive group of endometrial stromal tumors. A recent study described a recurrent chromosomal translocation $(t(10 ; 17))$ occurring in ESS, which joins the gene 14-3-3 $\varepsilon$ with either FAM22A or FAM22B. Expression of the resulting fusion gene leads to malignant transformation, and silencing of its expression reverses the malignant phenotype. Because the fusion can be readily detected in diagnostic samples using fluorescent in situ hybridization, this chromosomal aberration could be used to differentiate high-grade ESS from the low-grade, less aggressive form. Discovery of the new oncoprotein could also provide entry points for targeted therapies.
\end{abstract}

\section{Chromosomal translocations in cancer}

Chromosomal translocations that create gene fusions are a leading cause of malignant transformation in hematological malignancies and sarcomas [1]. Although they are less common in solid tumors, fusion genes are now also recognized as driving events in such tumors - for example, in prostate and thyroid cancers. Most of the cancer-causing translocations were detected by chromosome banding techniques [2], and they provided rewarding entry points for the positional cloning of oncogenes. Combined array comparative genomic hybridization and gene expression studies, as well as whole genome or exome sequence analysis, have now been added to the methods available to screen tumor genomes for recurrent aberrations, gene fusions or aberrant transcripts.

\footnotetext{
*Correspondence: riedt@mail.nih.gov, timo.gaiser@umm.de 'Genetics Branch, National Cancer Institute, National Institutes of Health, 50 South Drive, Bethesda, MD 20892, USA

2Department of Pathology, University Medicine Mannheim,

Theodor-Kutzer-Ufer 1-3, 68167 Mannheim, Germany
}

The prototypic example of a cancer-causing chromosomal translocation is the Philadelphia chromosome, first identified in patients with chronic myelogenous leukemia. The Philadelphia chromosome, generated through a reciprocal translocation between chromosomes 9 and 22 [3], results in a fusion transcript of the genes $B C R$ and $A B L 1$, which encodes a protein with increased tyrosine kinase activity. Patients with this translocation can be effectively treated with tyrosine kinase inhibitors, such as imatinib, which specifically targets the oncogenic fusion protein [4].

Endometrial stromal sarcoma (ESS) belongs to the group of endometrial stromal tumors, which are a category of relatively rare mesenchymal neoplasms of the uterus. The diagnostic spectrum of endometrial stromal tumors ranges from endometrial stromal nodule (ESN) through low-grade ESS (LESS) to high-grade (undifferentiated) ESS. ESNs are benign, well-circumscribed lesions, but LESS and ESS both show malignant behavior.

The distinction between LESS and ESS is clinically important because the prognosis of these lesions is drastically different. LESSs are indolent tumors with a low propensity for mainly local recurrences many years after surgical treatment. In contrast, ESSs are highly malignant and often develop extra-uterine metastases. The 5-year survival rate for LESS is close to $100 \%$, compared with 55\% for ESS [5]. The histological distinction is based on tumor cell necrosis, mitotic index and atypical cell morphology. However, both tumor types can show unusual features, resulting in difficulties for definitive tumor classification. Surgical removal of the fallopian tubes and ovaries on both sides remains the mainstay for the treatment of early stage disease and for LESS, whereas adjuvant or neo-adjuvant chemoradiotherapy is used for advanced stages and for ESS. However, a definitive benefit of chemoradiotherapy has yet to be established, highlighting the importance of targeted therapies [6].

\section{A novel mechanism in high-grade endometrial stromal sarcoma}

In a recently published study in the Proceedings of the National Academy of Sciences USA, Lee and colleagues 
[7] report a novel chromosomal aberration in this particularly aggressive form of uterine sarcoma. The chromosomal rearrangement identified is a translocation $t(10 ; 17)$, which fuses two genes: $14-3-3 \varepsilon$, which encodes a member of the 14-3-3 family (on chromosome 17), and either $F A M 22 A$ or FAM22B, both encoded on chromosome 10. The 14-3-3 family of proteins comprises seven members with numerous functions, including signal transduction, cytoskeletal configuration, metabolism, differentiation, survival and transcription [8]. Some of the members of this family are involved in tumorigenesis, either as suppressors or as oncogenes [9]. Very little is known about FAM22 proteins, and an involvement in malignant transformation has not been described before.

Lee et al. [7] performed a series of experiments to verify and characterize the chromosomal rearrangement. Whole-transcriptome sequencing showed that the fusion transcript was formed through joining of exon 5 of the $14-3-3 \varepsilon$ gene with exon 2 of either of the FAM22 genes. Fluorescent in situ hybridization analysis showed that the translocation is specific for the aggressive form of the uterine sarcomas and was not found in other mesenchymal tumors. The expression of the fusion protein was demonstrated by western-blot analysis in all translocation-positive tumors. Subsequently, the oncogenic properties of the fusion protein were explored by silencing the expression using RNA interference in a cell line derived from one of the tumors. This manipulation reduced the viability of the cell line, and overexpression of the fusion construct increased the migratory capacity of fibroblasts.

The authors [7] then sought to elucidate the functional properties of the fusion protein. Wild-type $14-3-3 \varepsilon$ is localized mainly in the cytoplasm, where it dimerizes. As a consequence of the translocation, abnormal nuclear localization of $14-3-3 \varepsilon$ protein was observed. This localization is possibly triggered by a nuclear localization sequence encoded by exon 7 of both FAM22A and FAM22B. The aberrant localization of a functional 14-3-3 protein to the nucleus most likely explains the transforming properties of the fusion protein.

It was already assumed that the inactivation of prominent tumor suppressors, including TP53, could explain the differences in tumor aggressiveness in ESS [10]. This view is now supported by comparing the gene expression of the translocation-positive higher-grade tumors with their low-grade counterparts. It became evident that genes involved in proliferation, such as CCND1 (encoding cyclin D1) and CEBPA (encoding CCAAT/enhancerbinding protein alpha), in addition to genes encoding factors that promote invasion, such as tissue inhibitor of metalloproteinase 1 (TIMP1) and fascin 1 (FSCN1), are more strongly expressed in the translocation positive high-grade tumors. CCND1, in particular, is a notorious oncoprotein that promotes cell cycle progression through cyclin-dependent-kinase-independent mechanisms.

\section{Clinical application in endometrial stromal sarcoma}

The immediate benefit of this study for the clinical management of patients with ESS is that the detection of the fusion protein greatly aids in diagnosing high-grade variants that may benefit from adjuvant chemoradiotherapy. Ideally, the identification of aberrant 14-3-3 signaling could be developed as a molecular therapeutic target, with the hope that evidence-based therapies will improve the outcome of patients with this aggressive cancer. Additional studies aimed at dissecting the precise role of the aberrant nuclear interaction that results from this translocation will undoubtedly identify downstream targets, which might also offer new therapeutic opportunities. The recent report by O'Meara and colleagues [11] that describes a 14-3-3-FAM22 fusion in a clear cell carcinoma of the kidney highlights the relevance of these particular cancer-inducing genes.

In summary, the paper by Lee et al. [7] presents an elegant example of a top-down strategy, where the identification of a specific and recurrent chromosomal translocation leads to the identification of a novel mechanism of deregulation of 14-3-3 family members, the identification of the genetic basis of a subgroup of tumors, the development of a novel, specific tool for differential diagnosis, and hopefully an entry point for targeted therapeutic strategies.

\section{Abbreviations}

CCND1, cyclin D1; ESN, endometrial stromal nodule; ESS, endometrial stromal sarcoma; LESS, low-grade endometrial stromal sarcoma.

\section{Competing interests}

The authors declare that they have no competing interests.

Published: 19 March 2012

\section{References}

1. Nussenzweig A, Nussenzweig MC: Origin of chromosomal translocations in lymphoid cancer. Cell 2010, 141:27-38.

2. Heim S, Mitelman F: Cancer Cytogenetics. Hoboken: John Wiley \& Sons; 2009.

3. Rowley JD: A new consistent chromosomal abnormality in chronic myelogeneous leukemia identified by quinacrine fluorescence and Giemsa staining. Nature 1973, 243:290-293.

4. Druker BJ: Translation of the Philadelphia chromosome into therapy for CML. Blood 2008, 112:4808-4817.

5. Norris HJ, Taylor HB: Mesenchymal tumors of the uterus. I. A clinical and pathological study of 53 endometrial stromal tumors. Cancer 1966, 19:755-766.

6. Amant F, Coosemans A, Debiec-Rychter M, Timmerman D, Vergote I: Clinical management of uterine sarcomas. Lancet Oncol 2009, 10:1188-1198.

7. Lee CH, Ou WB, Mariño-Enriquez A, Zhu M, Mayeda M, Wang Y, Guo X, Brunner AL, Amant F, French CA, West RB, McAlpine JN, Gilks CB, Yaffe MB, Prentice LM, McPherson A, Jones SJ, Marra MA, Shah SP, van de Rijn M, Huntsman DG, Dal Cin P, Debiec-Rychter M, Nucci MR, Fletcher JA: 14-3-3 fusion oncogenes in high-grade endometrial stromal sarcoma. Proc Natl Acad Sci U S A 2012, 109:929-934.

8. Aitken A: 14-3-3 proteins: a historic overview. Semin Cancer Bio/ 2006, 16:162-172. 
9. Hermeking $\mathrm{H}$ : The 14-3-3 cancer connection. Nat Rev Cancer 2003, 3:931-943.

10. Kurihara S, Oda Y, Ohishi Y, Iwasa A, Takahira T, Kaneki E, Kobayashi H, Wake N, Tsuneyoshi M: Endometrial stromal sarcomas and related high-grade sarcomas: immunohistochemical and molecular genetic study of 31 cases. Am J Surg Pathol 2008, 32:1228-1238.

11. O'Meara E, Stack D, Lee CH, Garvin AJ, Morris T, Argani P, Han JS, Karlsson J, Gisselson D, Leuschner I, Gessler M, Graf N, Fletcher JA, O'Sullivan MJ:
Characterization of the chromosomal translocation $\mathrm{t}(10 ; 17)(\mathrm{q} 22 ; \mathrm{p} 13)$ in clear cell sarcoma of kidney. J Patho/ 2012. doi: 10.1002/path.3985.

doi:10.1186/gm319

Cite this article as: Ried T, Gaiser T: A recurrent fusion gene in high-grade endometrial stromal sarcoma: a new tool for diagnosis and therapy?

Genome Medicine 2012, 4:20. 